\title{
CORRIGENDUM
}

\section{Long-term effect of exercise on bone mineral density and body composition in post-menopausal ex-elite athletes: a retrospective study}

\author{
A Andreoli ${ }^{1}, \mathrm{M} \mathrm{Celi}^{2}, \mathrm{SL}_{\text {Volpe }}{ }^{3}, \mathrm{R} \mathrm{Sorge}^{1,4}$ and U Tarantino ${ }^{2}$ \\ ${ }^{1}$ Human Physiology, University 'Tor Vergata', Rome, Italy; ${ }^{2}$ Department of Orthopaedics Surgery, University 'Tor Vergata', Rome, Italy; \\ ${ }^{3}$ Division of Biobehavioral and Health Sciences, School of Nursing, University of Pennsylvania, Philadelphia, PA, USA and ${ }^{4}$ Institute \\ of Medical Informatics and Biometry, University 'Tor Vergata', Rome, Italy
}

European Journal of Clinical Nutrition (2012) 66, 142; doi:10.1038/ejcn.2011.128

Correction to: European Journal of Clinical Nutrition advance online publication, 15 June 2011; doi:10.1038/ejcn.2011.104

Since the publication of this paper, the authors have noticed that the addresses for author affiliations 1 and 2 were incorrect. The correct list is shown above.
The authors apologise for any inconvenience caused. 